\title{
Research Paper \\ Effect of Bioflora and Cinnamon Extract Consumption on Dyslipidemia and Car- diovascular Disease in a Diabetic Rat Model
}

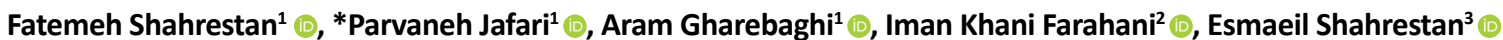

1. Department of Microbiology, Faculty of science, Islamic Azad University, Arak branch, Arak, Iran

2. Department of Materials Engineering, Faculty of Engineering, Shiraz University, Shiraz, Iran.

3. Department Physical Education and Sport Science, Brojerd Branch, Islamic Azad University, Brojerd, Iran.

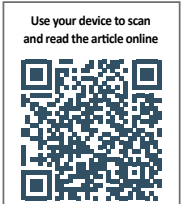

Citation: Shahrestan F, Jafari P, Khani I, Gharebaghi A, Shahrestan E. [Effect of Bioflora and Cinnamon Extract Consumption on Dyslipidemia and Cardiovascular Disease in a Diabetic Rat Model (Persian)]. Journal of Arak University of Medical Sciences (JAMS). 2020; 23(2):198-209. https://doi.org/10.32598/JAMS.23.2.5997.1

doi) https://doi.org/10.32598/JAMS.23.2.5997.1

Key words:

Diabetes, Cardiovascular disease, Dyslipidemia, Lipid profile, hs-CRP, Probiotic, Cinnamon extract

\section{A B S TRACT}

Article Info:

Available Online: 01 Jun 2020
Background and Aim Diabetes mellitus is one of the leading causes of death, and its prevalence is increasing annually because of the change in lifestyle. Increased blood glucose level and dyslipidemia are the major symptoms of this metabolic disease. Currently, the main and effective treatment for diabetes is the use of medication such as insulin. Its control by using herbal products has received a lot of attention in the world. The aim of this study is to evaluate the effects of bioflora (a probiotic supplement) and aqueous extract of cinnamon in improvement of blood glucose level, dyslipidemia, and reduction of cardiovascular diseases in diabetic rats.

Methods \& Materials Thirty-five male Wistar rats were prepared and randomly divided into five groups of negative control, positive control, probiotic (treated with bioflora $3.2 \times 108$ CFUs/day for 30 days), cinnamon (treated with $200 \mathrm{mg} / \mathrm{kg}$ of cinnamon aqueous extract for 30 days), and probiotic + cinnamon. Diabetes was induced by intra-peritoneally injection of streptozotocin. The rats' weight, blood glucose level, lipid profile, high sensitivity C-Reactive Protein (hs-CRP) and Atherogenic Index (Al) were measured at the end of experiment to predict the risk of cardiovascular diseases.

Ethical Considerations This study ethically approved in Research Centre of Islamic Azad University of Arak (Code: IR.IAU.ARAKREC1397.005). All interventions performed in accordance with the Guide for Care and Use of Laboratory Animals prepared by the Institute of Laboratory Animal Resources.

Results Induction of diabetes caused severe weight lost in rats, but the weight loss was lower in groups treated with probiotic. The blood glucose level in probiotic + cinnamon group was significantly reduced. Bioflora reduced triglyceride, cholesterol, and Low-Density Lipoprotein Cholesterol (LDL-C) levels, while cinnamon extract significantly reduced triglyceride and LDL-C levels compared to the positive control group. Al and hs-CRP values were reduced in the probiotic group compared to control groups. There was no synergistic effect by combined use of bioflora and cinnamon extract.

Conclusion Bioflora effectively prevented dyslipidemia by improving intestinal microbiota, lowering blood glucose level, and improving lipid profile and, therefore, reduced the risk of cardiovascular diseases.

\section{* Corresponding Author:}

\section{Parvaneh Jafari, PhD.}

Address: Department of Microbiology, Faculty of science, Islamic Azad University, Arak branch, Arak, Iran.

Tel: +98 (912) 2267223

E-mail: p-jafari@iau-arak.ac.ir 


\section{Extended Abstract}

\section{Introduction}

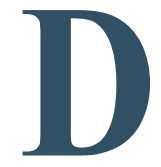

iabetes mellitus is an endocrine disease characterized by unusual lipid and sugar metabolisms. The global prevalence of diabetes has been rising rapidly. It is one of the leading causes of death in in low- and middle-income countries [2, 3]. There is an association between diabetes, metabolic syndrome and Cardiovascular Disease (CVD). Increased plasma level of Low-Density Lipoprotein (LDL) and Very-Low-Density Lipoprotein (VLDL) during metabolic syndrome, contribute to the pathogenesis of atherosclerosis [6-9]. Patients with diabetes are 2-4 times more at risk of CVD morbidity and mortality than individuals without diabetes. This study aimed to evaluate the effect of bioflora probiotic and aqueous extract of cinnamon plant on the amelioration of metabolic syndrome symptoms in rat model of diabetes. Bioflora is a commercial probiotic containing four probiotic strains include Lactobacillus acidophilus, Bifidobacterium longum, Bifidobacterium lactis, and Bifidobacterium bifidum.

\section{Materials and Methods}

In this study, 35 four-week-old male Wistar rats were purchased from Pasteur Institute of Iran. During the experiments, the animals were fed ad-libitum and had access to water all the times and kept under standard conditions.
After 10 days of adaptation, rats were randomly divided into 5 groups of negative control ( $\mathrm{n}=7$, healthy rats), positive control ( $\mathrm{n}=7$, untreated diabetic rats), probiotic group $(n=7$, diabetic rats treated with bioflora probiotic $3.2 \times 108$ CFUs/day), cinnamon group ( $\mathrm{n}=7$, diabetic rats treated with $200 \mathrm{mg} / \mathrm{kg}$ cinnamon aqueous extract daily), and probiotic + cinnamon $(\mathrm{n}=7)$. A single high dose $(60 \mathrm{mg} / \mathrm{kg}$ body weight) intra-peritoneal injection of Streptozotocin (STZ) was used to induce diabetes in rats. Only rats with a basal blood glucose level above $300 \mathrm{mg} / \mathrm{dL}$ were considered diabetic. After development of diabetes, rats were orally gavaged by $1 \mathrm{ml}$ suspension of probiotic, cinnamon aqueous extract, or both for four weeks. At the end of experiment, the animals were anesthetized and $5 \mathrm{ml}$ of blood samples were then extracted directly from their heart. The serum was separated from the clot by centrifugation and kept at $-20^{\circ} \mathrm{C}$. The glucose levels and lipid profile were assessed by using a specific assay kit (colorimetric). Quantitative immunoturbidimetric assay was used for identifying the high sensitivity C-Reactive Protein (hs-CRP) as a cardiovascular risk marker. Atherosclerosis Index (AI) was defined as log (Triglyceride/HDL-C).

\section{Results}

The STZ-induced diabetes caused a significant reduction in the body weight of animals where it was greater in animals treated with cinnamon aqueous extract (Table 1). The administration of bioflora probiotic increased the weight of rats in the probiotic group compared to other diabetic groups. It seems that the probiotic caused improvement in

Table 1. Mean levels of weight, blood glucose, lipid profile, AI and hs-CRP in different study groups

\begin{tabular}{|c|c|c|c|c|}
\hline \multirow{2}{*}{ Variables } & \multicolumn{4}{|c|}{ Mean $\pm S D$} \\
\hline & Weight (g) & Glucose (mg/dl) & Triglyceride (mg/dl) & LDL-C (mg/dl) \\
\hline Negative control & $21.33 \pm 3.48^{\mathrm{a}}$ & $168.3 \pm 00.49^{d}$ & $69.01+8.62^{\mathrm{bc}}$ & $13.97 \pm 2.53^{b}$ \\
\hline Positive control & $-28.50 \pm 3.50^{c}$ & $468.70 \pm 12.78^{a}$ & $119.00 \pm 6.49^{\mathrm{a}}$ & $26.88 \pm 1.69^{a}$ \\
\hline Probiotic & $-7.75 \pm 0.75^{b}$ & $329.05 \pm 8.19^{c}$ & $41.25 \pm 2.95^{c}$ & $17.00+1.02^{b}$ \\
\hline Cinnamon & $-29.01 \pm 2.00^{c}$ & $337.01 \pm 9.29^{b}$ & $71.33 \pm 8.51^{b}$ & $29.05 \pm 3.21^{\mathrm{a}}$ \\
\hline Probiotic + Cinnamon & $-11.96 \pm 2.14^{b}$ & $323.30 \pm 8.57^{c}$ & $41.33 \pm 4.06^{c}$ & $14.50 \pm 0.50^{\mathrm{b}}$ \\
\hline
\end{tabular}

\begin{tabular}{|c|c|c|c|c|}
\hline \multirow{2}{*}{ Variables } & \multicolumn{4}{|c|}{ Mean \pm SD } \\
\hline & HDL-C (mg/dl) & cholesterol (mg/dl) & hs-CRP (mg/lit) & Al \\
\hline Negative control & $23.25 \pm 1.44^{\mathrm{a}}$ & $56.50 \pm 4.91^{\mathrm{b}}$ & $2.76 \pm 0.11^{b}$ & $0.453 \pm 0.020^{b}$ \\
\hline Positive control & $23.33 \pm 0.88^{a}$ & $75.75 \pm 4.03^{\mathrm{a}}$ & $3.99 \pm 0.22^{\mathrm{a}}$ & $0.699 \pm 0.024^{\mathrm{ac}}$ \\
\hline Probiotic & $19.67 \pm 2.19^{\mathrm{a}}$ & $44.50+4.11^{d}$ & $2.53 \pm 0.16^{b}$ & $0.280 \pm 0.259^{d}$ \\
\hline Cinnamon & $18.05 \pm 2.08^{a}$ & $61.04 \pm 3.0^{\mathrm{ac}}$ & $4.08 \pm 0.71^{\mathrm{a}}$ & $0.598 \pm 0.071^{\mathrm{ac}}$ \\
\hline Probiotic + Cinnamon & $19.75 \pm 2.32^{\mathrm{a}}$ & $56.25 \pm 2.25^{c}$ & $3.04 \pm 0.27^{b}$ & $0.278 \pm 0.011^{\mathrm{d}}$ \\
\hline
\end{tabular}

Common letters in row shows there is no significant difference between value 
feed conversion ratio and feed uptake. Treatment with both bioflora probiotic and cinnamon extract led to a reduction in blood glucose, and also in cholesterol and triglyceride levels. The induction of diabetes had no any significant effect on the HDL-cholesterol (HDL-C) concentration. The LDLCholesterol (LDL-C) concentration, however, increased significantly with the diabetes induction. Bioflora probiotic reduced the LDL-cholesterol level while the cinnamon extract had no significant effect on this blood factor. The AI value was increased in rats due to bioflora probiotic administration. The hs-CRP, which is one the most important indicators of the risk of developing CVD, was increased in the positive control group. This factor significantly decreased in the probiotic group but its level in the cinnamon groups was not different from that in the positive control group. There was no reliable evidence of synergy between Bioflora probiotic and aqueous extract of cinnamon in amelioration of diabetes symptoms in rats.

\section{Discussion}

Insulin is a key hormone in glucose and lipid metabolisms. Insulin can regulate the production of a number of proteins affecting the circulating levels of lipoproteins. Insulin deficiency or insulin resistance in diabetes can lead to the development of dyslipidemia. Hence, people with diabetes are at high risk of CVDs. The results of this study showed that the blood glucose level in the groups treated with bioflora probiotic and cinnamon extract decreased by $19.65 \%$ and $29.81 \%$, respectively. The most important antidiabetic components of cinnamon are cinnamaldehyde, cinnamate, cinnamic acid, and eugenol. Procyanidin type $\mathrm{A}$ is an important polymer in cinnamon which increases the glucose absorption and glycogen synthesis in tissue while reduces the glucose uptake by intestinal epithelial cells [26, 27]. Some probiotic strains increase the glucagon gene expression and cause glucose hemostasis by upregulating the Peroxisome Proliferator Activated Receptor $\gamma$ (PPAR- $\gamma$ ) transcription factor [28-30].

The cinnamon extract improves lipid profile by inhibiting the $\beta$-hydroxy- $\beta$-methylglutaryl-coenzyme-A (HMG Co-A) reductase and reducing the oxidative stress. It is well documented that some bioflora probiotic strains can deconjugate the bile salts and lead to cholesterol reduction. The short-chain fatty acids produced by bioflora probiotic strains also inhibit the cholesterol synthesis in liver. Our results showed that bioflora reduced the risk of atherosclerosis by improvement of lipid profile. The hs-CRP level, as an indicator of CVD, is high in diabetic patients. The concentration of this factor increases 1000 times during infectious diseases and CVDs. Some strains of bioflora especially bifidobacteria, by inhibiting oxygen free radicals and reducing IL-6 gene expression, inhibit the increase of hsCRP and reduce inflammation. It was concluded that use of bioflora as supplement can prevent and improve metabolic syndrome in diabetic patients.

\section{Ethical Considerations}

\section{Compliance with ethical guidelines}

This study ethically approved in Research Centre of Islamic Azad University of Arak (Code: IR.IAU.ARAKREC1397.005). All interventions performed in accordance with the Guide for Care and Use of Laboratory Animals prepared by the Institute of Laboratory Animal Resources.

\section{Funding}

This article is extracted from a research project approved by research committee of Islamic Azad university of arak (Code: 266). Research deputy of this University financially suppoted part of this study.

\section{Authors' contributions}

Conceptualization: Parvaneh Jafari, Fatemeh Shahrestan; Methodology: Fatemeh Shahrestan, Aram Gharebaghi, Esmaeil Shahrestan; Data analysis: Parvaneh Jafari, Aram Gharebaghi; Investigation: All authors.

\section{Conflicts of interest}

The authors declared no conflict of interests.

\section{Acknowledgements}

The authors gratefully acknowledge the financial support of the Islamic Azad University of Arak and Tak Gene Company for kindy providing us with probiotic product. 


\title{
بهبود ديس ليبيدمى و كاهش خطر ابتلابه بيمارى هایى قلبى -عروقى با استفاده از يروبيوتيك بيوفلورا

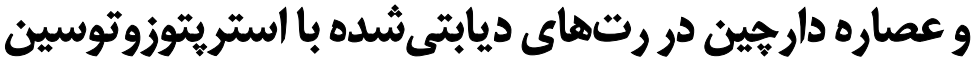

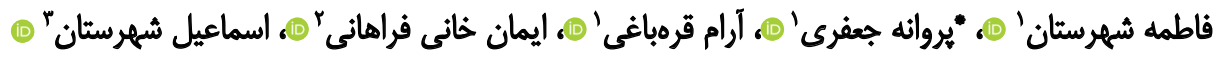 \\ 1. كروه ميكروبيولوزي، دانشكده علوم بايه، دانشكاه آزاد اسلامى، واحد اراك، اراك، ايران.

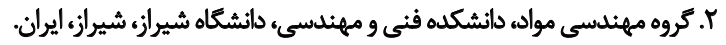

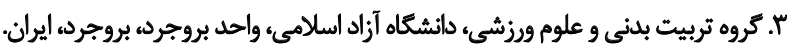

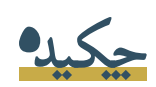

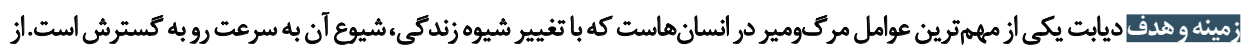

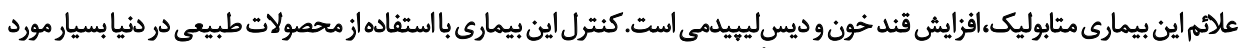

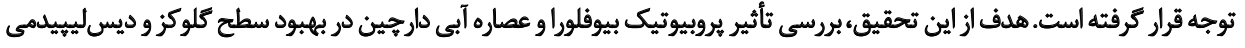

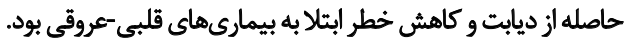

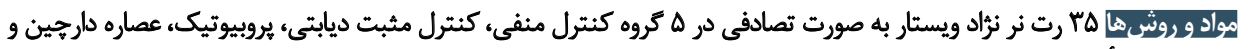

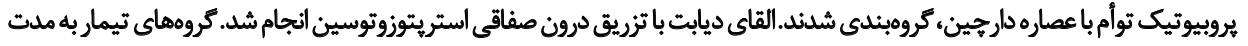

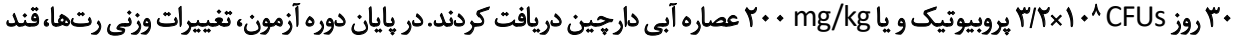

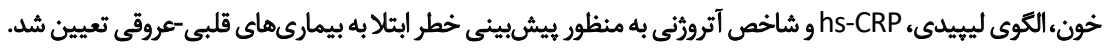

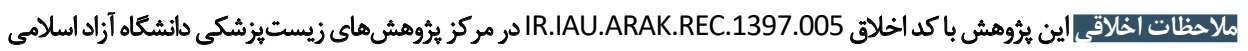
اراك به تصويب رسيده است.

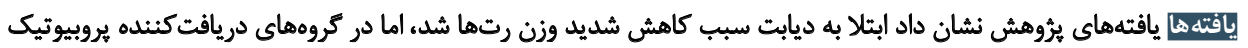

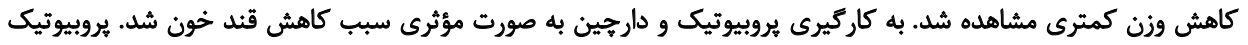

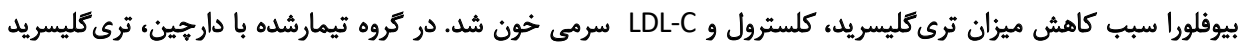

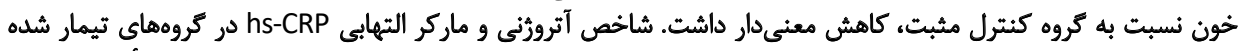

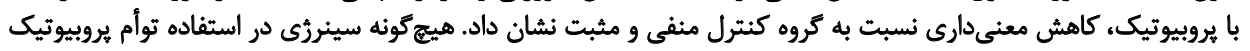

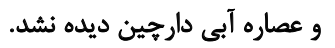

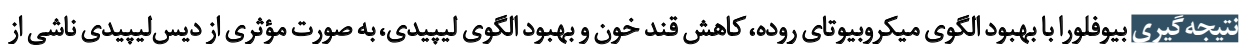

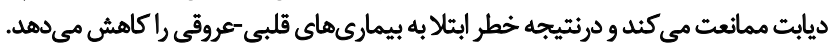

اطلاعات مقاله:

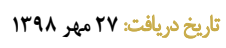

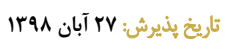
تاريخ انتشار: rاخرداد

كليدواروها:

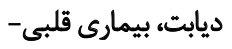

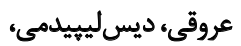
الكوى ليهيدى، يروتئين فئين

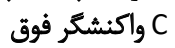
حساس، يروبيوتيك، عصاره دارجين ين يروني

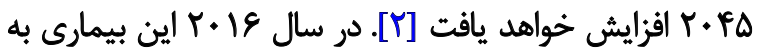

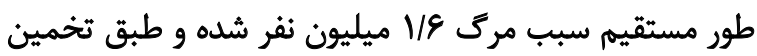

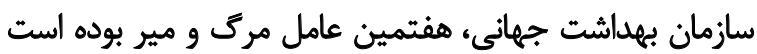

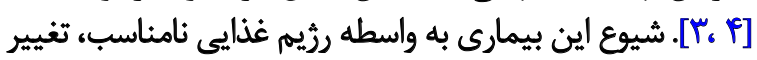

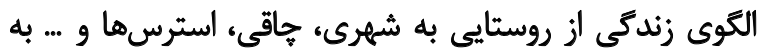

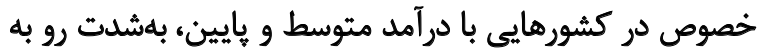

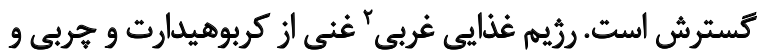

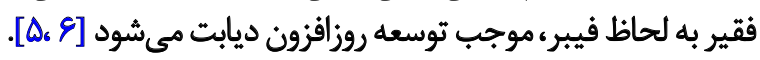

مقدمه

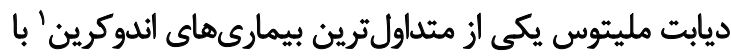

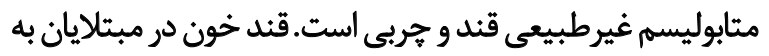

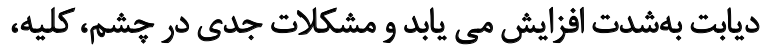

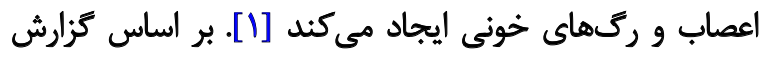

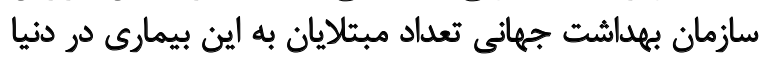

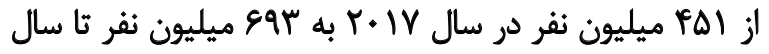


توليد ايمنوكلوبولين A و سايتوكاينهاي سركوبكر ايمنى، نقش

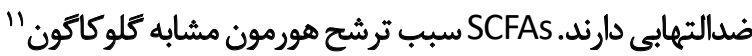

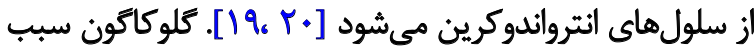

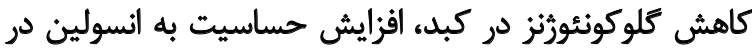

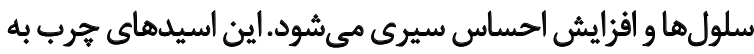

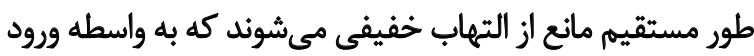

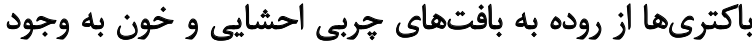

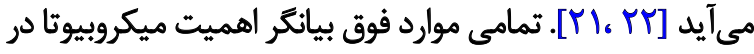

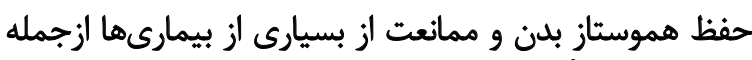

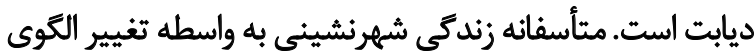

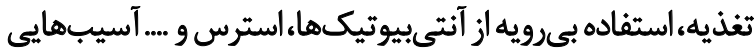

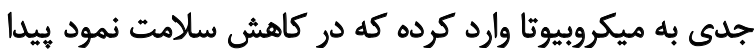

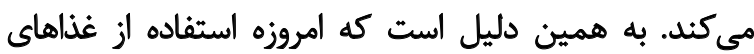

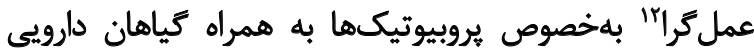

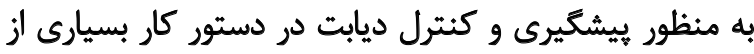

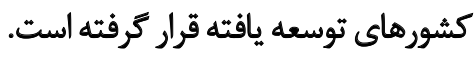

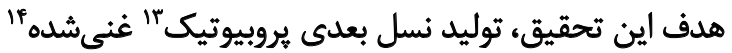

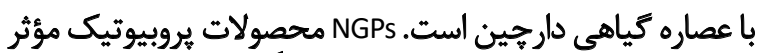

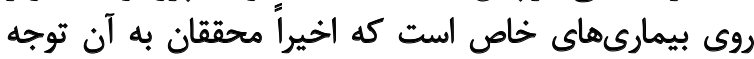

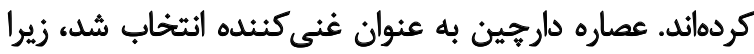

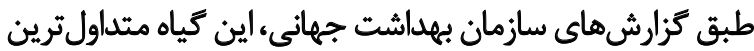

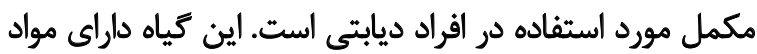

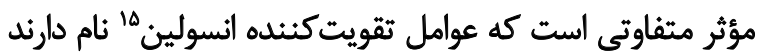

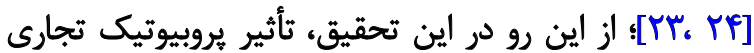

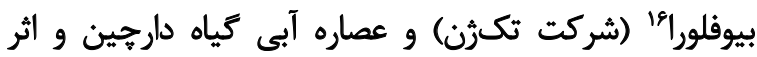

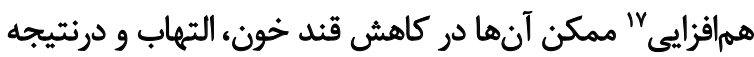

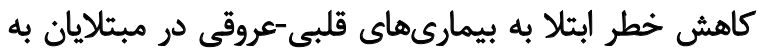

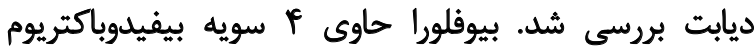

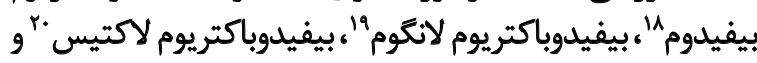

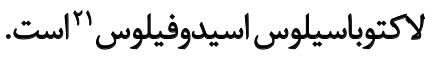

$$
\text { مواد و روش ها }
$$

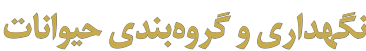

11.Glucagon like peptide-1

12.Functional Foods

13. Next Generation of Probiotic or NGP

14.Fortified

15. Insulin potentiating Factor

16.BioFlora

17.Synergism

18.B. bifidum

19.B. longum

20.B. lactis

21.L. acidophilus
در مبتلايان به ديابت، خطر ابتلا و مرك و مير به واسطه

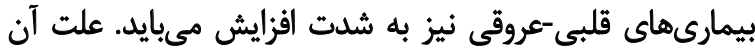

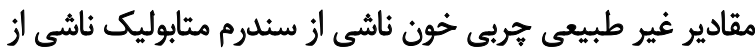

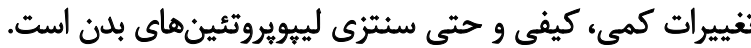

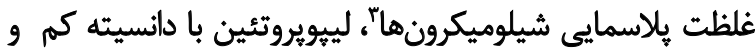
ليويروتئين با دانسيته بسيار كم (LDL)

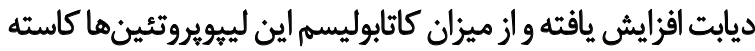

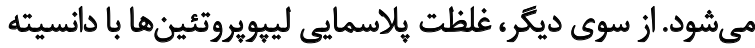

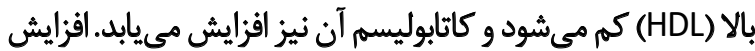

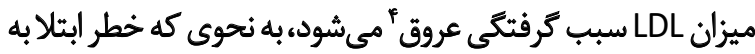

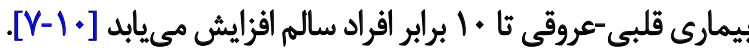
ديابت در ارتباط مستقيم با التهاب مزمن تحت بالينى هاست؛

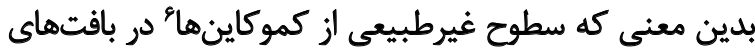

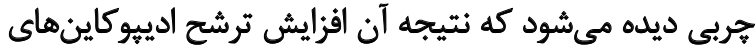

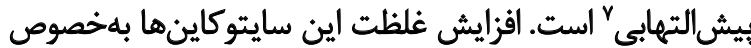

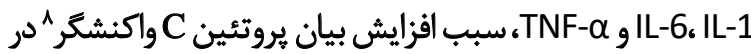

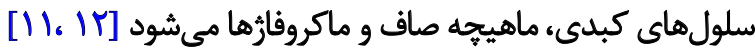

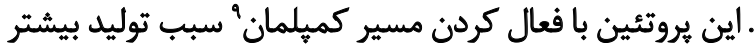

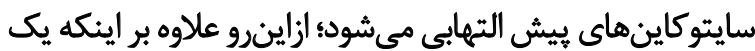

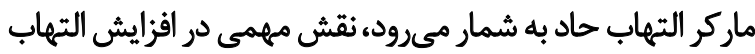

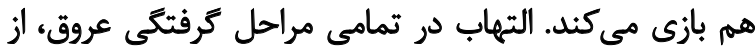

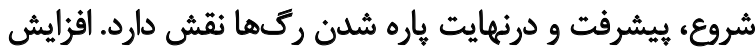

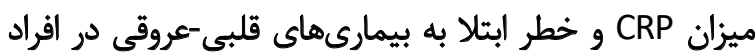

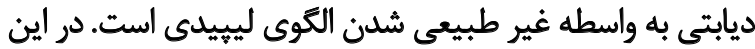

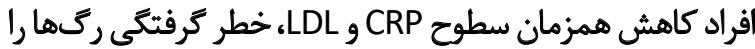

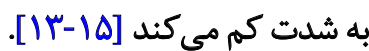

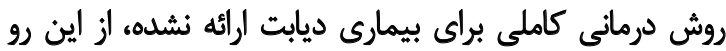

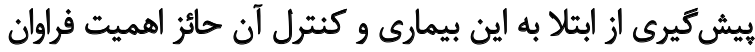

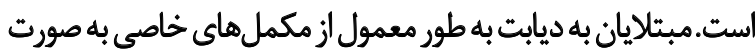

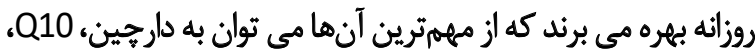

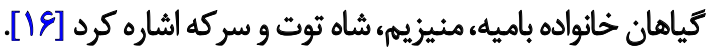
نقش ميكروبيوتاى روده در ممانعت از توسعه اين بيمارى، توجه

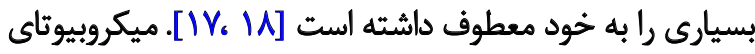

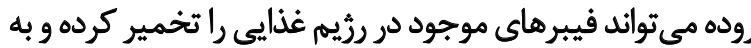
اسيدهاى جرب زنجيره كوثاه " تبديل كند. اين اسيدها باني تحريك

3.Chylomicrons

4.Atherosclerosis

5.Subclinical chronic inflammation

6.Chemokines

7.Pro inflammatory Adipokines

8.C Reactive Protein

9.Complement pathway

10.SCFA 


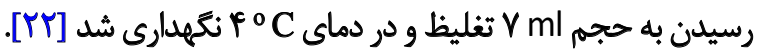

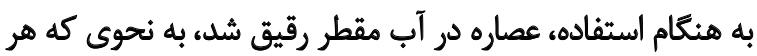

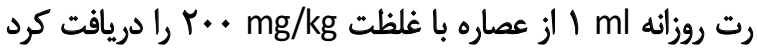

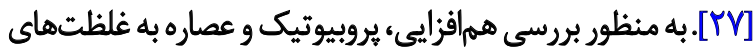

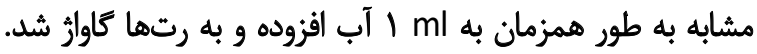

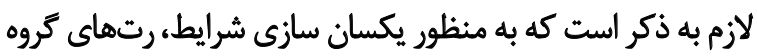

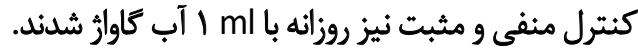

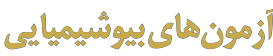

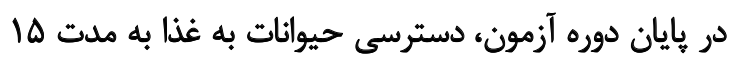

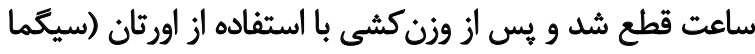

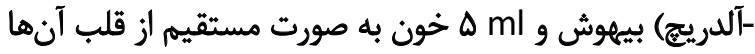

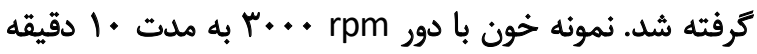

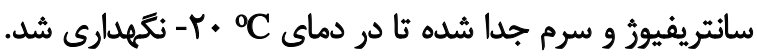

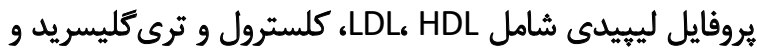

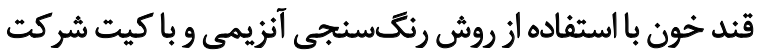

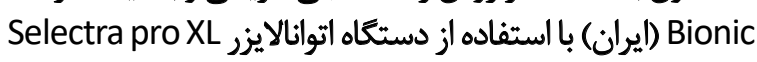

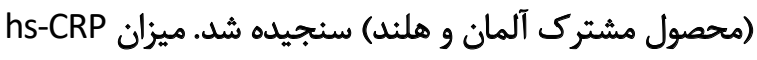

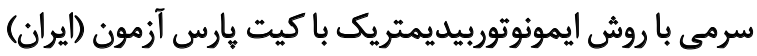

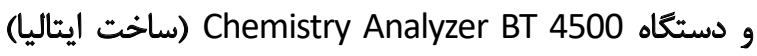
Atherogenic Index or) اندازه كرفته شد. شاخص آنالي)

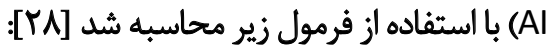

$$
\text { Atherogenic Index }=\log \left(\frac{\text { Triglyceride }}{\text { HDL-C }}\right)
$$

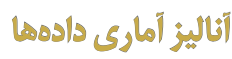

دادهها با استفاده از نرم افزار Graph pad prism6 و آزمونهاى

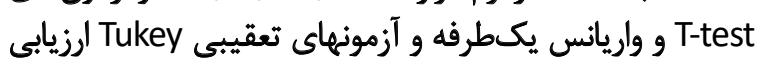

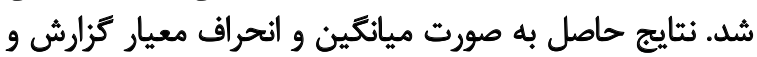

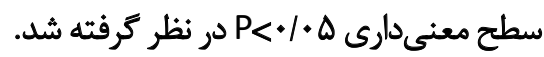

يافتهاها

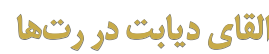

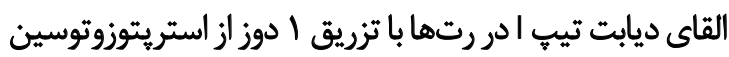

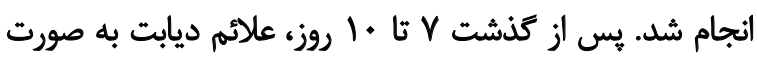

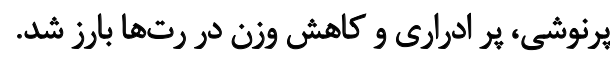

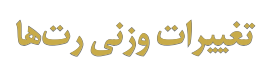

القاى ديابت سبب كاهش وزن معنى دارد روت رتها شد (جدول

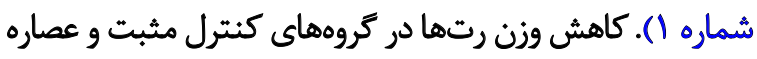

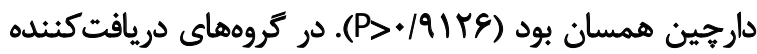

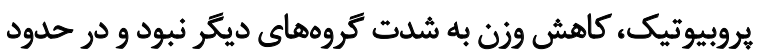

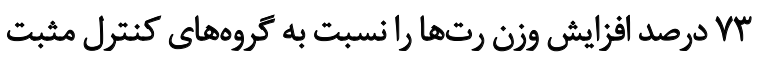

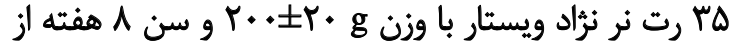

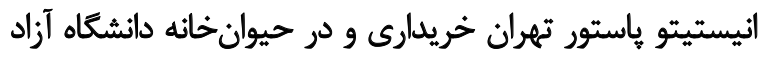

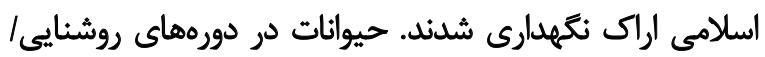

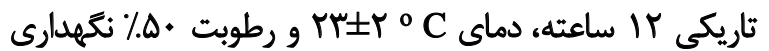

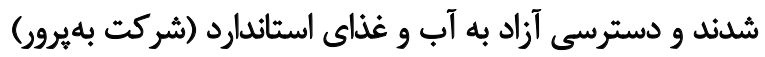

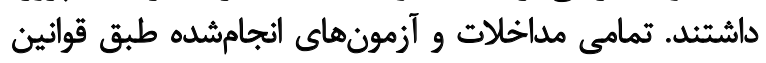

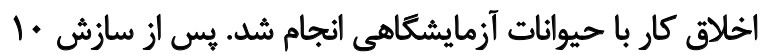
روزه، حيوانات به صورت تصادفى به له كروه تقسيم شدند:

$$
\text { كنترل منفى: رتهاى سالم }
$$

كنترل مثبت: رتهاى ديابتى بدون تيمار

كروه تجربى يروبيوتيك: رت ديابتى تيمار با بيوفلورا كروه تجربى دارجين: رت ديابتى تيمار با عصاره دارجين

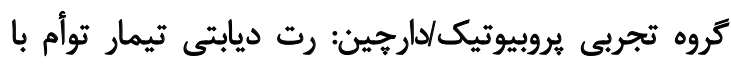

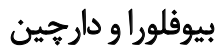

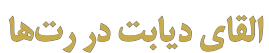

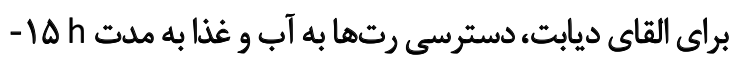

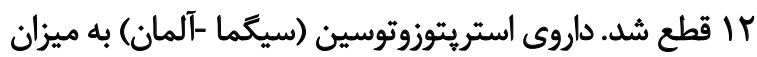

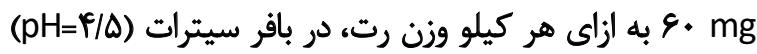

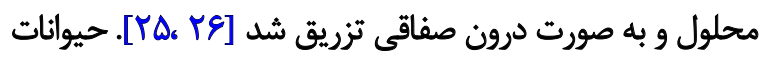

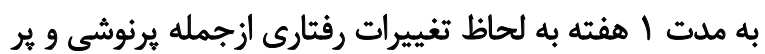

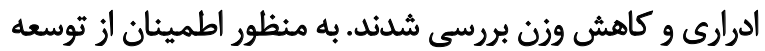

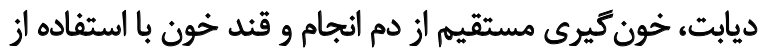

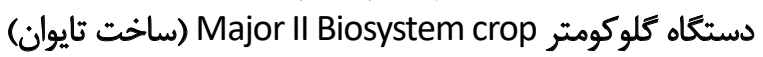

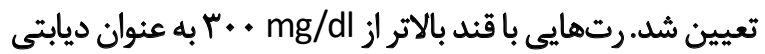

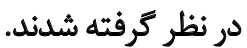

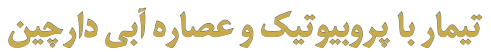

محتويات هر كيسول بيوفلورا شركت تكثرن (//) ميليارد

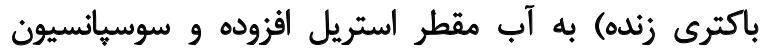

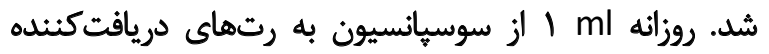

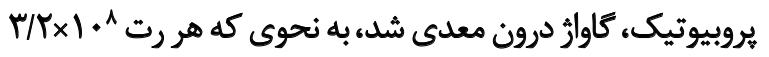

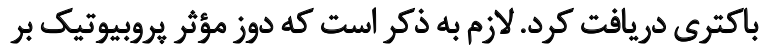

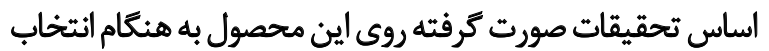

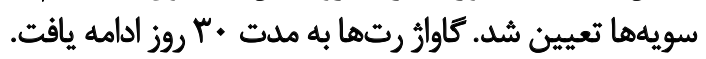
Cinnamomum Zeyl-) براي تهيه عصاره آبى، نمونه دارجين از مركز ملى ذخاير ايران تهيه و از سوى هربارين إيوم دانشكاه (anicum

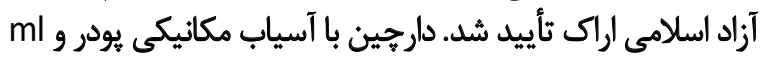

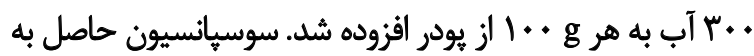

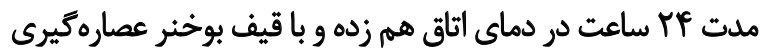

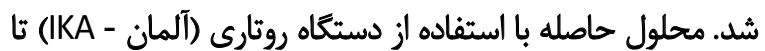


جدول ا. ميانكين و انحراف معيار ميزان وزن كيرى، كلوكز سرمى، يروتئ C واكنشكَر فوقحساس در كروههاي مختلف آزمون

\begin{tabular}{|c|c|c|c|c|}
\hline $\mathrm{Al}$ & Hs-CRP (mg/lit) & (mg/dl) (كلوكز & وزنكيرى (g) & كروه ها \\
\hline$. / F \Delta r \pm+/ .199 b$ & $T / N G \pm \cdot / I^{b}$ & $\mid S N / N^{\prime}+. / / 9 q^{d}$ & $r / / r+\Psi / F \Lambda^{a}$ & كنترل منفى \\
\hline 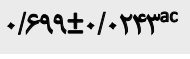 & $. / r Y \pm r / 99^{a}$ & $F E N V \cdot \pm I Y / N \lambda^{a}$ & $-r N \Delta \cdot \pm r / \Delta \cdot{ }^{C}$ & كتترل مثبت \\
\hline$\cdot / R A \cdot \pm \cdot / T \Delta Q^{b}$ & $r / \Delta r \pm . / / \& b$ & $r \mathrm{rq} / \Delta \pm N 19^{\circ}$ & $-V / N \Delta \pm \cdot N \Delta^{b}$ & يروبيوتيك \\
\hline$\cdot / \Delta q \pm \pm . \cdot v \mid r^{\mathrm{ac}}$ & $\varphi / \cdot A \pm \cdot(N)^{a}$ & $m W / \cdot 1 \pm q / q^{b}$ & $-r q \cdot 1 \pm Y / . .0$ & مارجين \\
\hline$. / r V A \pm . / .11 r^{\mathrm{d}}$ & $r / \cdot f \pm \cdot / r^{b}$ & $M \pi / r+ \pm N \Delta \gamma^{C}$ & $-11 / Q \& \pm T / / e^{b}$ & هيرو+دارجين \\
\hline
\end{tabular}

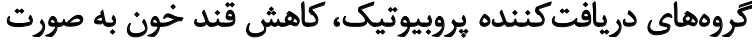

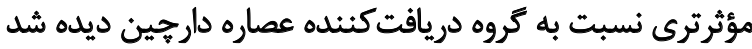

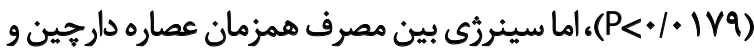

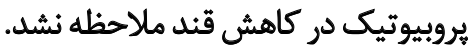

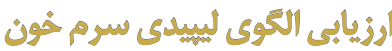

القاى ديابت در رتها سبب افزايش معنى دار كلسترول و

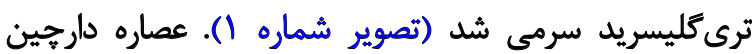

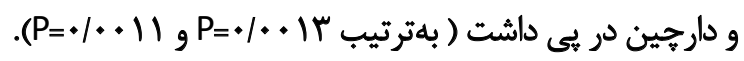$$
\text { كاهش قند خون سرمى با يروبيوتيك و عصاره دارجين }
$$

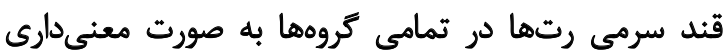

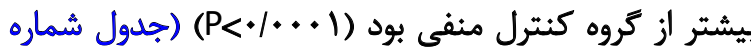

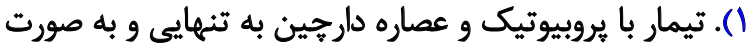

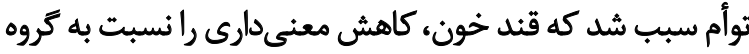

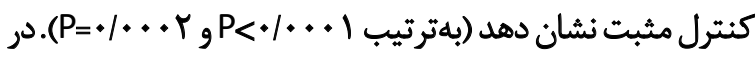
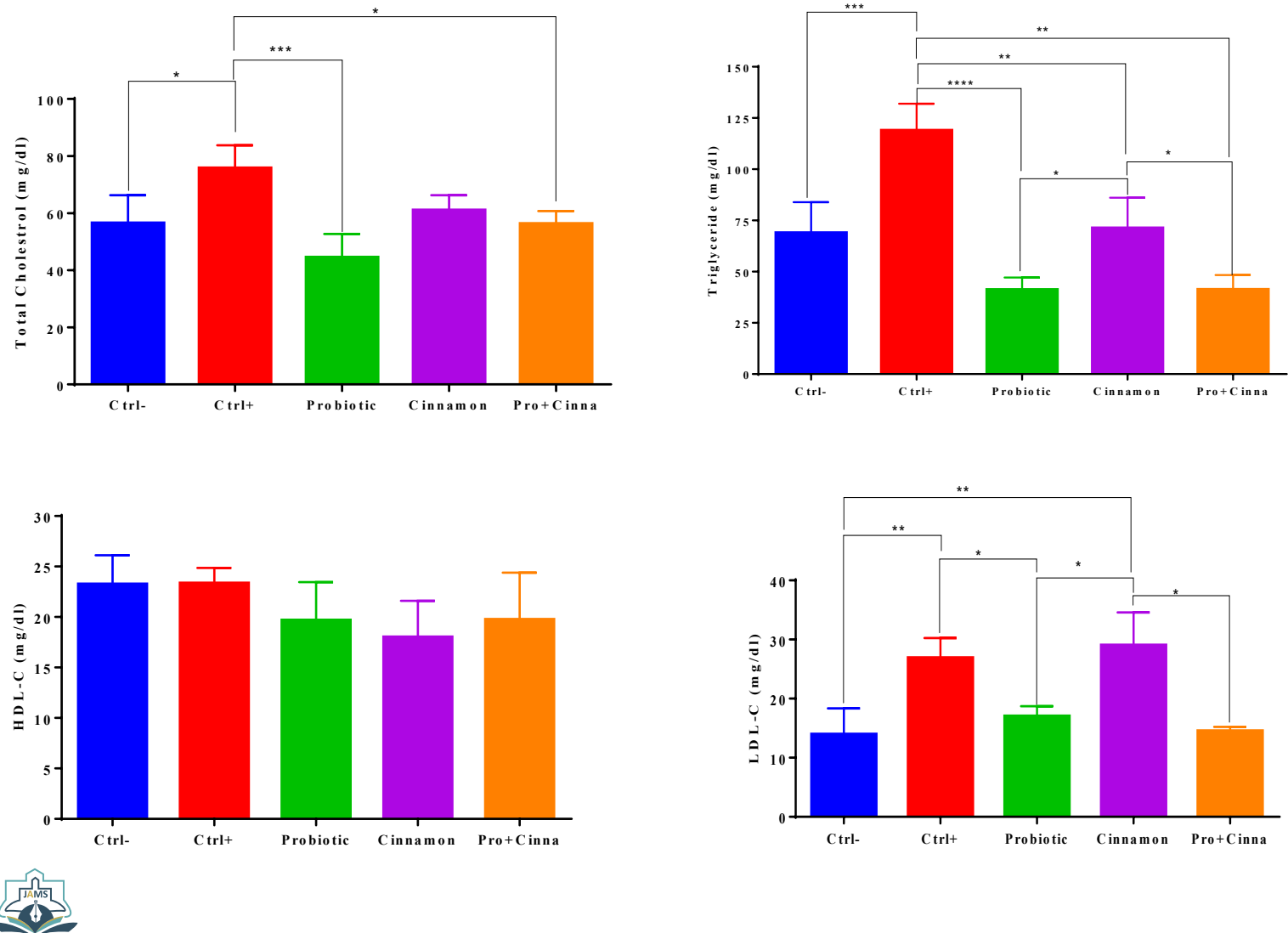

تصوير ا. ميزان ترى كليسريد، كلسترول ثام، C-LDL , C-LDH در كروههاي مختلف رته هيس از يك ماه تيمار. نمودارهاي نمايشكر ميانكين و خطاى استاندارد ميانكين (Mean士SEM) هر كروه است.

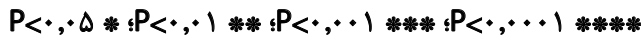




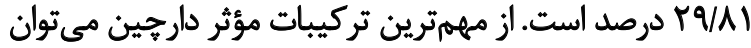

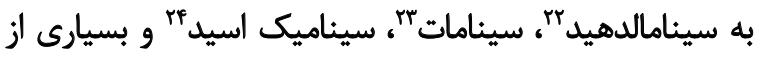

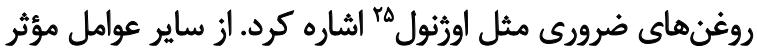

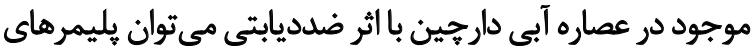

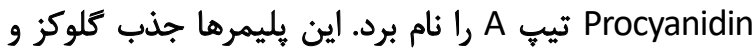

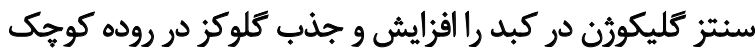

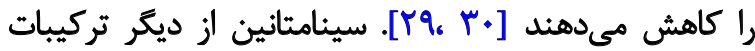

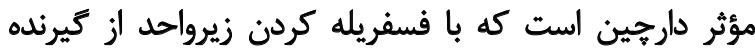

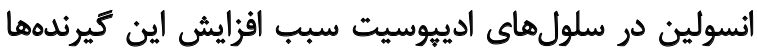

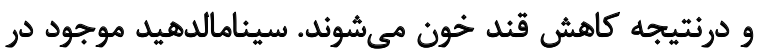

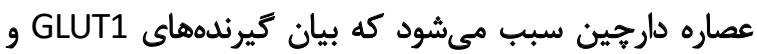

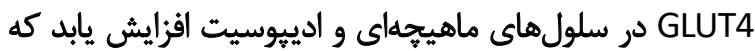

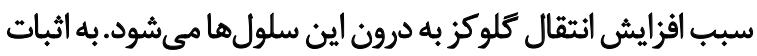

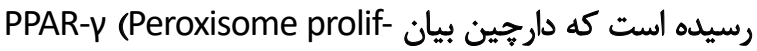

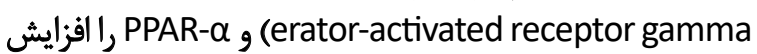
مىدهد. بيان اين يروتئينها سبب افزايش حساسيت سلولها به

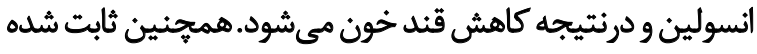

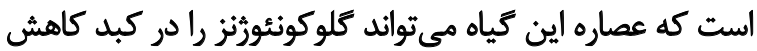

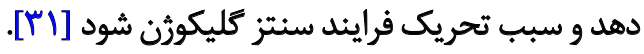

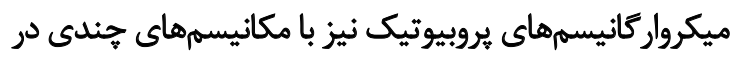

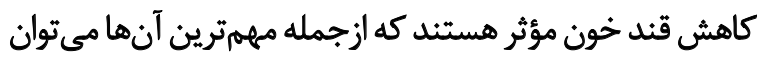

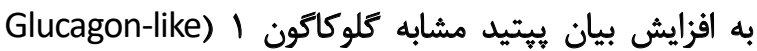

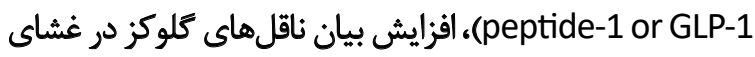
سلولى و افزايش بيان PPAR- اشاره كرد كه فاكتور رونويسى

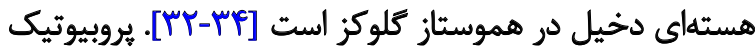

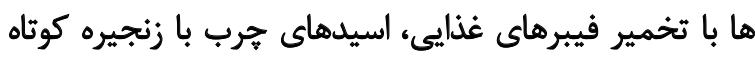

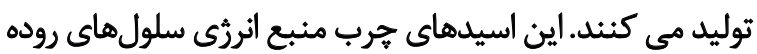

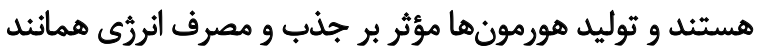

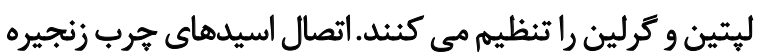

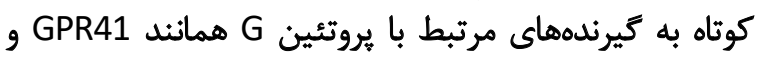

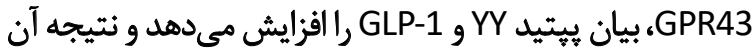

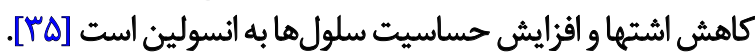

نتايج نشان داد كه در شرايط به كار كرفته در اين تحقيق،

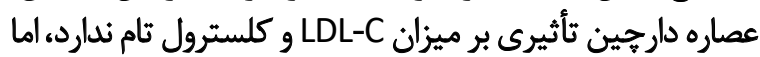

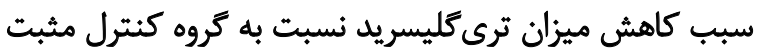

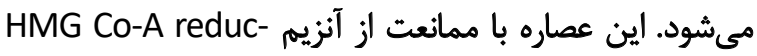

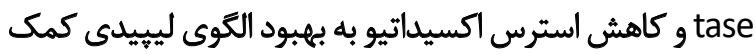

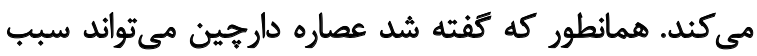
افزايش بيان PPAR- شود كه تنظيمكنينده تمايز سلولهن

22.Cinnamaldehyde

23. Cinnamate

24.Cinnamic acid

25.Eugenol
به صورت معنى دارى سبب كاهش ترى كليسريد در رتهاي

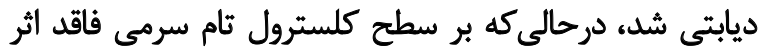

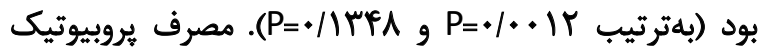

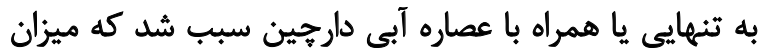

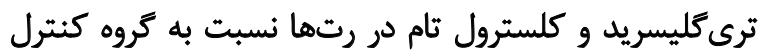

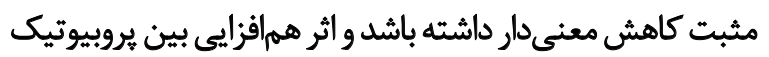

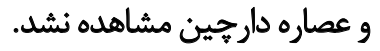

بررسى سطوح HDL-C نشان داد كه اختلالات متابوليسمى

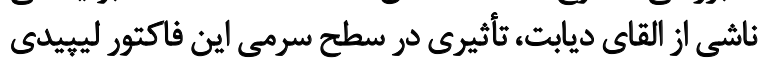

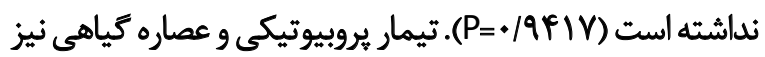
تغييرى در غلظت سرمى اين فاكتور خونى ايجاد نكرد، در صورتى ني

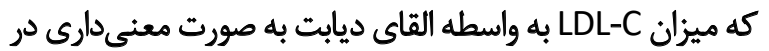

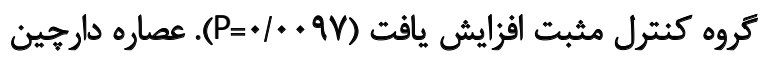

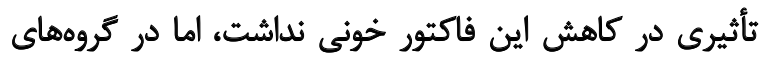

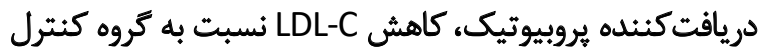

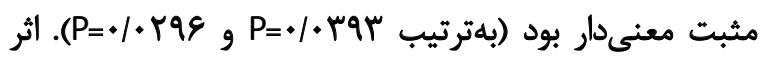

هم|فزايى نيز در به كاركيرى همزمان اين دو مشاهده نشد.

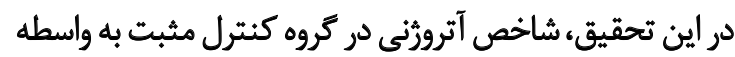

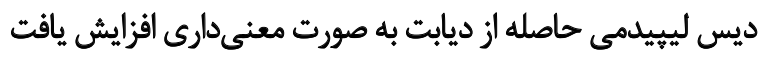

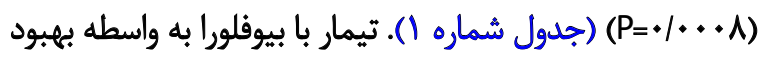

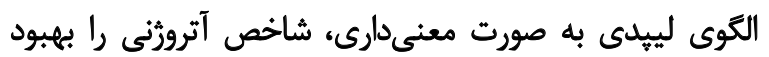

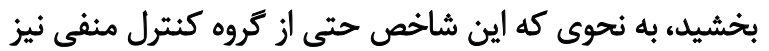

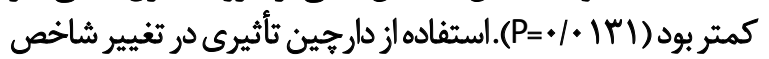

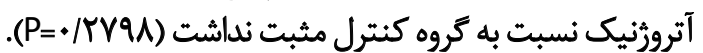

\section{hs-CRP بورسي سطوح}

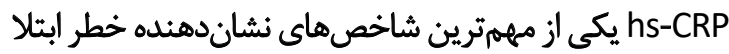

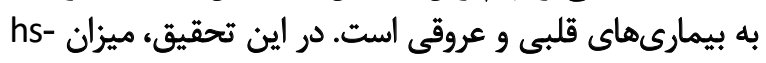

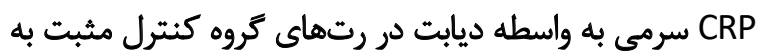

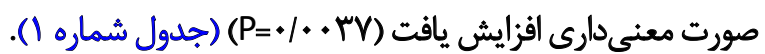

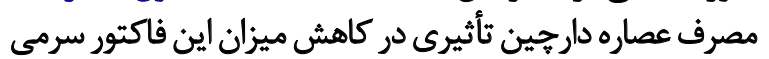

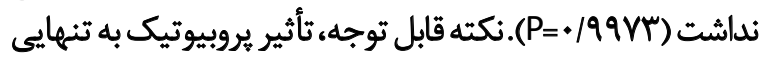

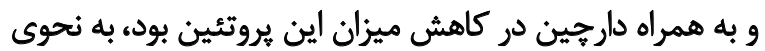

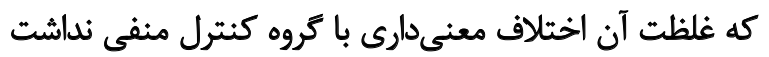

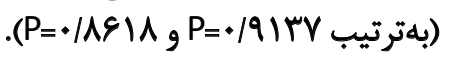

\section{تئيجلمَيرى}

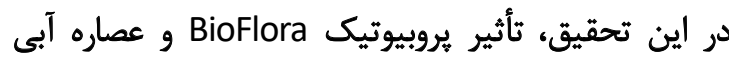

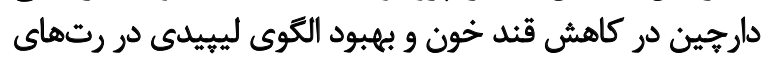

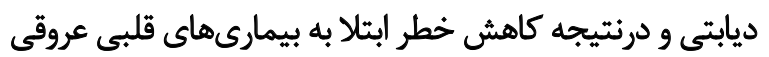

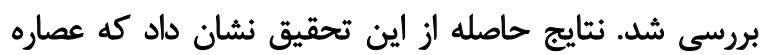

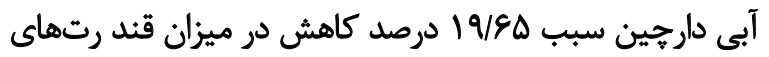
ديابتى مي دوده، در حالى كه در كروه يروبيوتيك اين مقدار دار معاد 
ديسلييدمى حاصل از ديابت را بهبود بخشد. لازم به ذكر است كه

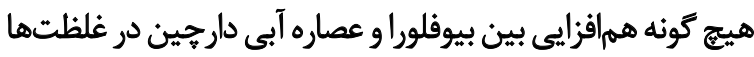
و مدت زمان به كار كرفته شده در اين تحقيق وجود ندارئ داردا.

مالاحظات اخلاقي

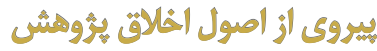

ايـن بروهـش بـا كـد اخـلاق بـه شـماره IRIAU.ARAK ه.REC

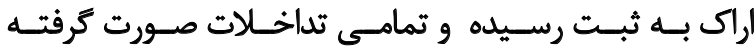

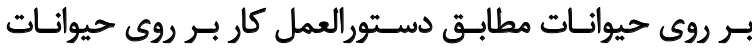
آزمايشـكاهى بـوده اسـت

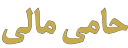

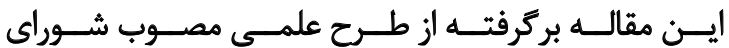

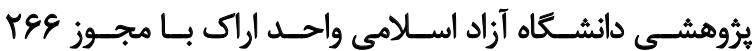

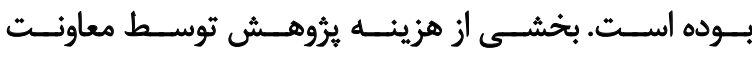

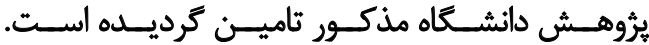

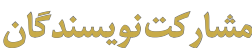

تعريف موضوع و بيان مسئله :دكتر بروانه جعفرى، فاطمه

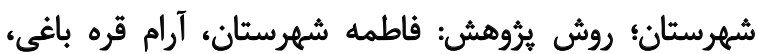

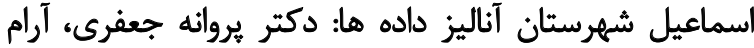

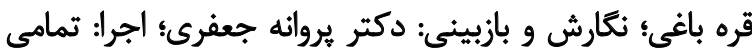

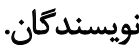

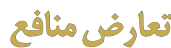

نويسندكان تصريح مي كنئد كه هيجگتونه تضاد منافعى در

خصوص يثوهش حاضر وجود ندارد.

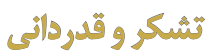

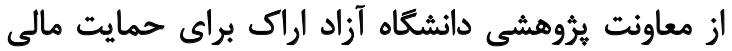

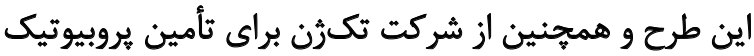

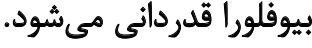

ادييوسيت محسوب مي شود. ثابت شده است كه فعال شدن اين

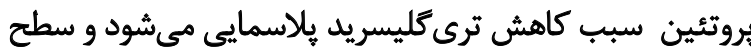
تر HDL

تحقيق نيز مشاهده شد [اهن]

دمر رتهاي كروه يروبيوتيك، ميزان كلسترول تام، LDL-C

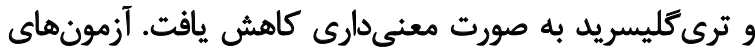

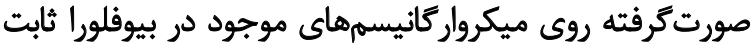

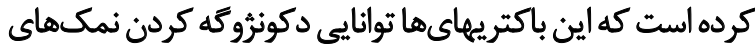

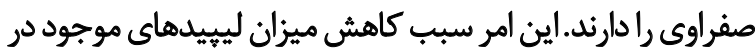

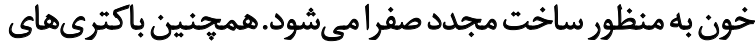

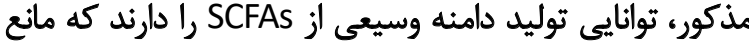

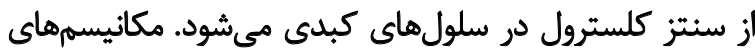

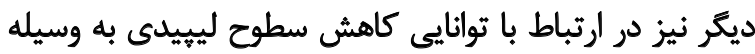

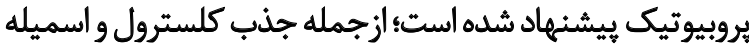

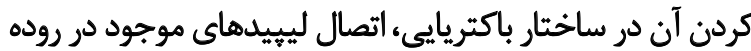

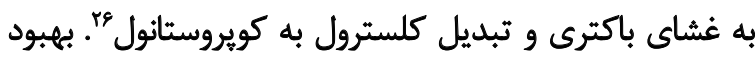

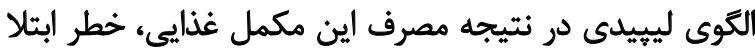

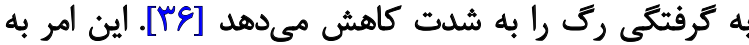

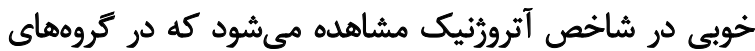

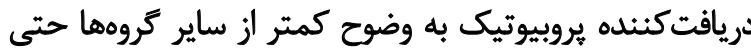

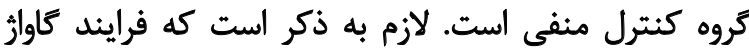

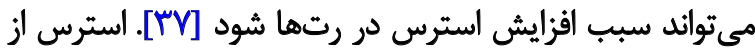

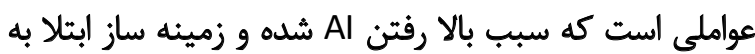

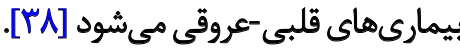

در اين تحقيق، كاهش خطر ابتلا به بيمارى قلبى -عروقى با باديات

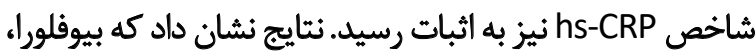

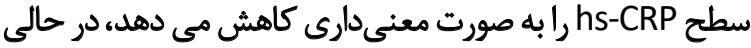

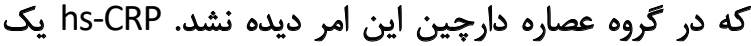

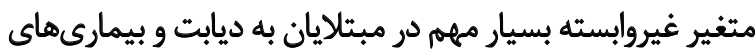

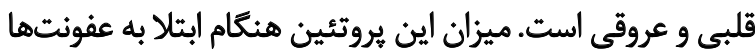

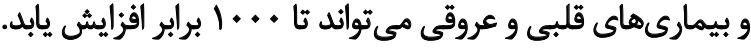

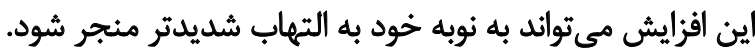

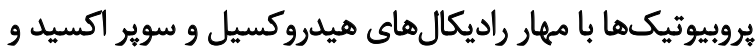

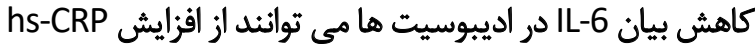
جلوكيرى كنند و التهاب را كاهش دهند.

بحث

عصاره دارجين و بيوفلورا هر دو خطر ابتلا به بيمارىهاي قلبى كائي

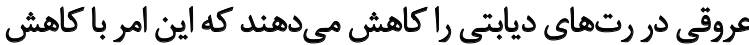

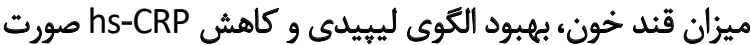

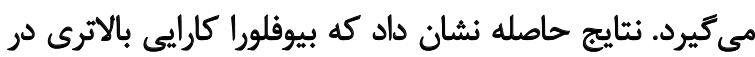

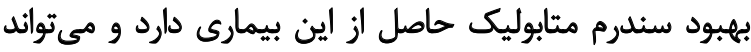

26.Coprostanol 


\section{References}

[1] Lavin N. Manual of endocrinology and metabolism. Philadelphia, Pennsylvania, United States: Lippincott Williams \& Wilkins; 2012.

[2] Cho N, Shaw J, Karuranga S, Huang Y, Da Rocha Fernandes J, Ohlrogge A, et al. IDF Diabetes Atlas: Global estimates of diabetes prevalence for 2017 and projections for 2045. Diabetes Research and Clinical Practice. 2018; 138:271-81. [DOI:10.1016/j.diabres.2018.02.023] [PMID]

[3] Whiting DR, Guariguata L, Weil C, Shaw JJDr, practice c. IDF diabetes atlas: global estimates of the prevalence of diabetes for 2011 and 2030. Diabetes Research and Clinical Practice. 2011; 94(3):311-21. [DOI:10.1016/j.diabres.2011.10.029] [PMID]

[4] Balakumar P, Maung UK, Jagadeesh G J P R. Prevalence and prevention of cardiovascular disease and diabetes mellitus. Pharmacological Research. 2016; 113 (Part A):600-9. [DOI:10.1016/j. phrs.2016.09.040] [PMID]

[5] Pérez-Martínez P, García-Ríos A, Delgado-Lista J, Pérez-Jiménez F, LópezMiranda JJ. Mediterranean diet rich in olive oil and obesity, metabolic syndrome and diabetes mellitus. Current Pharmaceutical Design. 2011; 17(8):769-77. [DOI:10.2174/138161211795428948] [PMID]

[6] Guariguata L, Whiting DR, Hambleton I, Beagley J, Linnenkamp U, Shaw JEJDR, et al. Global estimates of diabetes prevalence for 2013 and projections for 2035. Diabetes Research and Clinical Practice. 2014; 103(2):137-49. [DOI:10.1016/j.diabres.2013.11.002] [PMID]

[7] Klop B, Elte J, Cabezas MJN. Dyslipidemia in obesity: Mechanisms and potential targets. Nutrients. 2013; 5(4):1218-40. [DOI:10.3390/ nu5041218] [PMID] [PMCID]

[8] Wu L, Parhofer KGJM. Diabetic dyslipidemia. Diabetes Therapy. 2014; 63(12):1469-79. [DOI:10.1016/j.metabol.2014.08.010] [PMID]

[9] Vergès BJD. Pathophysiology of diabetic dyslipidemia: Where are we? Diabetologia. 2015; 58(5):886-99. [DOI:10.1007/ s00125-015-3525-8] [PMID] [PMCID]

[10] Jaiswal M, Schinske A, Pop-Busui RJBP, Endocrinology RC, Metabolism. Lipids and lipid management in diabetes. Best Practice \& Research: Clinical Endocrinology. 2014; 28(3):325-38. [DOI:10.1016/j. beem.2013.12.001] [PMID]

[11] Esser N, Legrand-Poels S, Piette J, Scheen AJ, Paquot NJDR. Inflammation as a link between obesity, metabolic syndrome and type 2 diabetes. Diabetes Research and Clinical Practice. 2014; 105(2):141-50. [DOI:10.1016/j.diabres.2014.04.006] [PMID]

[12] Mahluji S, Ostadrahimi A, Mobasseri M, Attari VE, Payahoo LA. Antiinflammatory effects of Zingiber officinale in type 2 diabetic patients. Advanced Pharmaceutical Bulletin. 2013; 3(2):273.

[13] Laakso MJDc. Cardiovascular disease in type 2 diabetes from population to man to mechanisms: The Kelly West Award Lecture 2008. Diabetes Care. 2010; 33(2):442-9. [DOI:10.2337/dc09-0749] [PMID] [PMCID]

[14] Lee SH, Park SA, Ko SH, Yim HW, Ahn YB, Yoon KH, et al. Insulin resistance and inflammation may have an additional role in the link between cystatin $C$ and cardiovascular disease in type 2 diabetes mellitus patients. Metabolism. 2010; 59(2):241-6. [DOI:10.1016/j.metabol.2009.07.019] [PMID]

[15] Parrinello CM, Lutsey PL, Ballantyne CM, Folsom AR, Pankow JS, Selvin EJAhJ. Six-year change in high-sensitivity C-reactive protein and risk of diabetes, cardiovascular disease, and mortality. American Heart Journal. 2015; 170(2):380-9. [DOI:10.1016/j.ahj.2015.04.017] [PMID] [PMCID]
[16] Ahmadi E, Alizadeh-Navaei R, Rezai MS. Efficacy of probiotic use in acute rotavirus diarrhea in children: A systematic review and meta-analysis. Caspian Journal of Internal Medicine. 2015; 6(4):187-95.

[17] Parekh PJ, Nayi VR, Johnson DA, Vinik AlJFie. The role of gut microflora and the cholinergic anti-inflammatory neuroendocrine system in diabetes mellitus. Frontiers in Endocrinology. 2016; 7:55. [DOI:10.3389/ fendo.2016.00055] [PMID] [PMCID]

[18] Hartstra AV, Bouter KE, Bäckhed F, Nieuwdorp M. Insights into the role of the microbiome in obesity and type 2 diabetes. Diabetes Care. 2015 38(1):159-65. [DOI:10.2337/dc14-0769] [PMID]

[19] Tolhurst G, Heffron H, Lam YS, Parker HE, Habib AM, Diakogiannaki E, et al. Short-chain fatty acids stimulate glucagon-like peptide-1 secretion via the G-protein-coupled receptor FFAR2. Diabetes. 2012; 61(2):36471. [DOI:10.2337/db11-1019] [PMID] [PMCID]

[20] Kim CH, Park J, Kim MJIn. Gut microbiota-derived short-chain fatty acids, T cells, and inflammation. Immune Network. 2014; 14(6):277-88. [DOI:10.4110/in.2014.14.6.277] [PMID] [PMCID]

[21] Koh A, De Vadder F, Kovatcheva-Datchary P, Bäckhed F. From dietary fiber to host physiology: short-chain fatty acids as key bacterial metabolites. Cell. 2016; 165(6):1332-45. [DOI:10.1016/j. cell.2016.05.041] [PMID]

[22] Blaut MJPotNS. Gut microbiota and energy balance: role in obesity. Proceedings of the Nutrition Society. 2015; 74(3):227-34. [DOI:10.1017/ S0029665114001700] [PMID]

[23] Qin B, Panickar KS, Anderson RA. Cinnamon: Potential role in the prevention of insulin resistance, metabolic syndrome, and type 2 diabetes. Journal of Diabetes Science and Technology. 2010; 4(3):685-93. [DOI:10 1177/193229681000400324] [PMID] [PMCID]

[24] Gruenwald J, Freder J, Armbruester N. Cinnamon and health. Critical Reviews in Food Science and Nutrition. 2010; 50(9):822-34. [DOI:10.1080/10408390902773052] [PMID]

[25] Babujanarthanam R, Kavitha P, Rao UM, Pandian MR. Quercitrin a bioflavonoid improves the antioxidant status in streptozotocin: Induced diabetic rat tissues. Fundamental \& Clinical Pharmacology. 2011; 358(12):121. [DOI:10.1007/s11010-011-0927-x] [PMID]

[26] Etuk EJABJA. Animals models for studying diabetes mellitus. Agriculture and Biology Journal of North America. 2010; 1(2):130-4.

[27] Shokri G, Fathi H, Jafari Sabet M, Nasri Nasrabadi N, Ataee R. Evaluation of anti-diabetic effects of hydroalcoholic extract of green tea and cinnamon on streptozotocin-induced diabetic rats. 2015, 1(2):20-9. [DOI:10.18869/acadpub.pbr.1.2.20]

[28] Dobiášová M, Frohlich J, Šedová M, Cheung MC, Brown BG. Cholesterol esterification and atherogenic index of plasma correlate with lipoprotein size and findings on coronary angiography. Journal of Lipid Research. 2011; 52(3):566-71. [DOI:10.1194/jlr.P011668] [PMID] [PMCID]

[29] Mollazadeh $\mathrm{H}$, Hosseinzadeh HJljobms. Cinnamon effects on metabolic syndrome: a review based on its mechanisms. 2016;19(12):1258

[30] Lu Z, Jia Q, Wang R, Wu X, Wu Y, Huang C, Li Y. Hypoglycemic activities of $\mathrm{A}$-and $\mathrm{B}$-type procyanidin oligomer-rich extracts from different Cinnamon barks. Phytomedicine. 2011; 18(4):298-302. [DOI:10.1016/j. phymed.2010.08.008] [PMID]

[31] Medagama ABJ. The glycaemic outcomes of Cinnamon, a review of the experimental evidence and clinical trials. Nutrition Journal. 2015; 14(1):108. [DOI:10.1186/s12937-015-0098-9] [PMID] [PMCID] 
[32] Shah NJ, Swami OC. Role of probiotics in diabetes: A review of their rationale and efficacy. 2017.

[33] Aw W, Fukuda Sh. Understanding the role of the gut ecosystem in diabetes mellitus. Journal of Diabetes Investigation. 2018; 9(1):5-12. [DOI:10.1111/jdi.12673] [PMID] [PMCID]

[34] Mishra AK, Dubey V, Ghosh AR. Obesity: An overview of possible role (s) of gut hormones, lipid sensing and gut microbiota. Metabolism. 2016; 65(1):48-65. [DOI:10.1016/j.metabol.2015.10.008] [PMID]

[35] Ebrahimi FS, Rad AH, Mosen M, Abbasalizadeh F, Tabrizi A, Khaliil L Effect of $L$. acidophilus and $B$. lactis on blood glucose in women with gestational diabetes mellitus: A randomized placebo-controlled trial. Diabetology \& Metabolic Syndrome. 2019; 11(1):75. [DOI:10.1186/ s13098-019-0471-5] [PMID] [PMCID]

[36] Gadelha CJMU, Bezerra ANJJvb. Effects of probiotics on the lipid profile: Systematic review. Jornal Vascular Brasileiro. 2019; 18:e20180124.

[37] Brown AP, Dinger N, Levine BS. Stress produced by gavage administration in the rat. Contemporary topics in laboratory animal science/ American Association for Laboratory Animal Science. 2000; 39(1):17-21.

[38] Yao BC, Meng LB, Hao ML, Zhang YM, Gong T, Guo Z-gJJolMR. Chronic stress: a critical risk factor for atherosclerosis. Journal of International Medical Research. 2019; 47(4):1429-40. [DOI:10.1177/0300060519826820] [PMID] [PMCID] 
This Page Intentionally Left Blank 